AAPS PharmSciTech 2005; 6 (1) Article 10 (http://www.aapspharmscitech.org).

\title{
Formulation and Evaluation of Mucoadhesive Glipizide Microspheres
}

Submitted: July 16, 2004; Accepted: November 12, 2004; Published: September 20, 2005

Jayvadan K. Patel, ${ }^{1}$ Rakesh P. Patel, ${ }^{1}$ Avani F Amin, ${ }^{2}$ and Madhabhai M. Patel ${ }^{1}$

${ }^{1}$ Shree S.K. Patel College of Pharmaceutical Education and Research, Ganpat Vidyanagar, Mehsana-Gozaria Highway, Kherva-382711, India

${ }^{2}$ Nirma Institute of Pharmacy, Nirma University of Science and Technology, Sarkhej-Gandhinagar Highway,

Ahmedabad-380061, India

\section{Abstract}

The purpose of this research was to formulate and systematically evaluate in vitro and in vivo performances of mucoadhesive microspheres of glipizide. Glipizide microspheres containing chitosan were prepared by simple emulsification phase separation technique using glutaraldehyde as a cross-linking agent. Results of preliminary trials indicate that volume of cross-linking agent, time for cross-linking, polymer-to-drug ratio, and speed of rotation affected characteristics of microspheres. Microspheres were discrete, spherical, and free flowing. The microspheres exhibited good mucoadhesive property in the in vitro wash-off test and also showed a high percentage drug entrapment efficiency. A $3^{2}$ full factorial design was employed to study the effect of independent variables, polymer-to-drug ratio $\left(X_{1}\right)$, and stirring speed $\left(X_{2}\right)$ on dependent variables percentage mucoadhesion, $\mathrm{t}_{80}$, drug entrapment efficiency, and swelling index. The best batch exhibited a high drug entrapment efficiency of $75 \%$ and a swelling index of 1.42 ; percentage mucoadhesion after 1 hour was $78 \%$. The drug release was also sustained for more than 12 hours. The polymer-to-drug ratio had a more significant effect on the dependent variables. In vivo testing of the mucoadhesive microspheres to albino Wistar rats demonstrated significant hypoglycemic effect of glipizide.

KEYWORDS: chitosan, mucoadhesive microspheres, glipizide, factorial design, in vivo study

\section{INTRODUCTION}

Microsphere carrier systems made from the naturally occurring biodegradable polymers have attracted considerable attention for several years in sustained drug delivery. Recently, dosage forms that can precisely control the release rates and target drugs to a specific body site have made an enormous impact in the formulation and develop-

Corresponding Author: Jayvadan K. Patel, Shree S.K. Patel College of Pharmaceutical Education and Research, Ganpat Vidyanagar, Mehsana-Gozaria Highway, Kherva382711, India. Tel: 0091-2762-286082; Fax: 0091-2762286082.E-mail: jayvadan04@yahoo.com ment of novel drug delivery systems. Microspheres form an important part of such novel drug delivery systems. ${ }^{1-3}$ They have varied applications and are prepared using assorted polymers. ${ }^{4}$ However, the success of these microspheres is limited owing to their short residence time at the site of absorption. It would, therefore, be advantageous to have means for providing an intimate contact of the drug delivery system with the absorbing membranes. ${ }^{5-8}$ This can be achieved by coupling bioadhesion characteristics to microspheres and developing bioadhesive microspheres. Bioadhesive microspheres have advantages such as efficient absorption and enhanced bioavailability of drugs owing to a high surface-to-volume ratio, a much more intimate contact with the mucus layer, and specific targeting of drugs to the absorption site. ${ }^{9-12}$ Chitosan (obtained by deacetylation of chitin) is a cationic polymer that has been proposed for use in microsphere systems by a number of authors. ${ }^{13-17}$ Chitosan was selected as a polymer in the preparation of mucoadhesive microspheres because of its good mucoadhesive and biodegradable properties.

Glipizide is a second-generation sulfonylurea that can acutely lower the blood glucose level in humans by stimulating the release of insulin from the pancreas and is typically prescribed to treat type II diabetes (non-insulindependent diabetes mellitus). Its short biological half-life ( $3.4 \pm 0.7$ hours) necessitates that it be administered in 2 or 3 doses of 2.5 to $10 \mathrm{mg}$ per day. ${ }^{18}$ Thus, the development of controlled-release dosage forms would clearly be advantageous. Researchers have formulated oral controlled-release products of glipizide by various techniques. ${ }^{12,19,20}$ Moreover, the site of absorption of glipizide is in the stomach. Dosage forms that are retained in the stomach would increase the absorption, improve drug efficiency, and decrease dose requirements. Thus, an attempt was made in this investigation to use chitosan as a mucoadhesive polymer and prepare microspheres. The microspheres were characterized by in vitro and in vivo tests, and factorial design was used to optimize the variables.

\section{MATERIALS AND MethodS}

\section{Materials}

Glipizide was obtained as gift sample from USV Ltd (Daman, India). Chitosan (degree of deacetylation of 85\%; 
AAPS PharmSciTech 2005; 6 (1) Article 10 (http://www.aapspharmscitech.org).

Table 1. Result of Preliminary Trial Batches*

\begin{tabular}{|c|c|c|c|c|c|}
\hline Batch Code & $\begin{array}{c}\text { Volume of } \\
\text { Glutaraldehyde } \\
(\mathrm{mL})\end{array}$ & $\begin{array}{c}\text { Cross-linking } \\
\text { Time (h) }\end{array}$ & $\begin{array}{c}\text { In Vitro Wash-off Test } \\
\text { (\% Mucoadhesion } \\
\text { After } 1 \text { h) }\end{array}$ & $\begin{array}{c}\text { Drug Entrapment } \\
\text { Efficiency }(\%)\end{array}$ & $\begin{array}{l}\text { Sphericity of } \\
\text { Microspheres }\end{array}$ \\
\hline $\mathrm{J} 1$ & 10 & 1 & 85 & $38( \pm 0.6)$ & Very irregular \\
\hline $\mathrm{J} 2$ & 10 & 2 & 79 & $40( \pm 1.2)$ & \\
\hline $\mathrm{J} 3$ & 10 & 3 & 74 & $42( \pm 1.9)$ & \\
\hline $\mathrm{J} 4$ & 20 & 1 & 81 & $52( \pm 2.1)$ & Slightly irregular \\
\hline $\mathrm{J} 5$ & 20 & 2 & 75 & $57( \pm 1.3)$ & \\
\hline J6 & 20 & 3 & 68 & $59( \pm 0.8)$ & \\
\hline $\mathrm{J} 7$ & 40 & 1 & 72 & $58( \pm 2.4)$ & Spherical free flowing \\
\hline $\mathrm{J} 8$ & 40 & 2 & 66 & $60( \pm 1.1)$ & \\
\hline J9 & 40 & 3 & 59 & $62( \pm 0.8)$ & \\
\hline $\mathrm{J} 10$ & 50 & 1 & 74 & $68( \pm 2.1)$ & Spherical free flowing \\
\hline $\mathrm{J} 11$ & 50 & 2 & 65 & $70( \pm 1.1)$ & \\
\hline $\mathrm{J} 12$ & 50 & 3 & 60 & $74( \pm 1.8)$ & \\
\hline $\mathrm{J} 13$ & 60 & 1 & 61 & $67( \pm 2.1)$ & Spherical free flowing \\
\hline $\mathrm{J} 14$ & 60 & 2 & 54 & $70( \pm 1.4)$ & \\
\hline $\mathrm{J} 15$ & 60 & 3 & 47 & $69( \pm 0.5)$ & \\
\hline
\end{tabular}

*All the batches were prepared at a polymer-to-drug ratio of $3: 1$.

intrinsic viscosity, $1390 \mathrm{~mL} / \mathrm{g}$ in $0.30 \mathrm{M}$ acetic acid $/ 0.2 \mathrm{M}$ sodium acetate solution; and viscometric molecular weight, $4.08 \times 10^{5} \mathrm{Da}$ ) was obtained as gift sample from Central Institute of Fisheries Technology (Cochin, India). Dioctyl sodium sulfosuccinate (DOSS) and petroleum ether 80:20 were procured from Willson Lab (Mumbai, India) and S. D. Fine Chemicals Ltd (Mumbai, India), respectively. Liquid paraffin and glutaraldehyde were purchased from Loba Chemie Pvt Ltd (Mumbai, India).

\section{Methods}

\section{Preparation of Microspheres}

Mucoadhesive microspheres of chitosan were prepared by simple emulsification phase separation technique. Chitosan was used as a polymer and was cross-linked using glutaraldehyde as per method described by Thanoo et al. ${ }^{13}$

Chitosan $(1.5 \mathrm{~g})$ was dissolved in $150 \mathrm{~mL}$ of $1 \% \mathrm{vol} / \mathrm{vol}$ aqueous acetic acid solution. Five hundred milligrams of drug was dispersed in the polymer solution. In batches J1 to J15 the polymer-to-drug ratio was kept constant at 3:1. The resultant mixture was extruded through a syringe (No. 20 ) in $1 \mathrm{~L}$ of liquid paraffin (heavy and light, 1:1 ratio) containing $0.2 \%$ DOSS, and stirring was performed using a propeller stirrer (Remi, Mumbai, India) at $1000 \mathrm{rpm}$. After 15 minutes, glutaraldehyde ( $25 \% \mathrm{vol} / \mathrm{vol}$ aqueous solution) was added and stirring was continued. The amount of cross-linking agent and cross-linking time were varied in batches $\mathrm{J} 1$ to $\mathrm{J} 15$ from 10 to $60 \mathrm{~mL}$ and 1 to 3 hours, respectively, as shown in Table 1. In factorial design batches $\mathrm{B} 1$ to $\mathrm{B} 9,50 \mathrm{~mL}$ of glutaraldehyde was used as a cross- linking agent and cross-linking time was kept to 1 hour. The polymer-to-drug ratio and stirring speed were varied in batches B1 to B9 as shown in Table 2. All other variables were used as mentioned in preliminary trial batches. Microspheres thus obtained were filtered and washed several times with petroleum ether $(80: 20)$ to remove traces of oil. They were finally washed with water to remove excess of glutaraldehyde. The microspheres were then dried at room temperature (at $25^{\circ} \mathrm{C}$ and $60 \%$ relative humidity [RH]) for 24 hours. The effect of formulation variables on characteristics of the microspheres is summarized in Tables 1 and 2.

\section{Assay of Glipizide}

Glipizide was estimated by ultraviolet visible (UV/Vis) spectrophotometric method (Shimadzu UV-1601 UV/Vis double beam spectrophotometer, Kyoto, Japan). Aqueous solutions of glipizide were prepared in phosphate buffer (pH 7.4) and absorbance was measured on UV/Vis spectrophotometer at $276 \mathrm{~nm} .^{21}$ The method was validated for linearity, accuracy, and precision. The method obeys Beer's Law in the concentration range of 5 to $50 \mu \mathrm{g} / \mathrm{mL}$. When a standard drug solution was analyzed repeatedly $(\mathrm{n}=5)$, the mean error (accuracy) and relative standard deviation (precision) were found to be $0.8 \%$ and $1.3 \%$, respectively.

\section{Drug Entrapment Efficiency}

Microspheres $(50 \mathrm{mg}$ ) were crushed in a glass mortar and pestle, and the powdered microspheres were suspended in 
Table 2. $3^{2}$ Full Factorial Design Layout*

Variable Levels in Coded Form
In Vitro Wash-off Test

(\% Mucoadhesion After 1 h)

\begin{tabular}{ccccc} 
fter 1 h) & tso (minutes) & $\begin{array}{c}\text { Drug Entrapment } \\
\text { Efficiency }(\%)\end{array}$ & $\begin{array}{c}\text { Swelling } \\
\text { Index }\end{array}$ & $\begin{array}{c}\text { Particle } \\
\text { Size }(\boldsymbol{\mu m})\end{array}$ \\
\hline 51 & 236 & 52 & 0.866 & 60.4 \\
45 & 234 & 50 & 0.819 & 56.7 \\
42 & 220 & 47 & 0.808 & 49.3 \\
74 & 207 & 70 & 1.172 & 67.1 \\
65 & 239 & 68 & 1.113 & 64.9 \\
59 & 250 & 64 & 0.982 & 61.6 \\
78 & 476 & 75 & 1.423 & 96.0 \\
71 & 456 & 71 & 1.282 & 88.2 \\
65 & 380 & 68 & 1.229 & 71.8
\end{tabular}

Translation of Coded Levels in Actual Units

\begin{tabular}{lccc}
\hline Variables Level & Low $(-1)$ & Medium $(0)$ & High $(+1)$ \\
\hline Polymer-to-drug ratio $\left(X_{1}\right)$ & $1: 1$ & $3: 1$ & $6: 1$ \\
Stirring speed $\left(X_{2}\right)$ rpm & 500 & 1000 & 1500 \\
\hline
\end{tabular}

*All the batches were prepared using $50 \mathrm{~mL}$ of glutaraldehyde and a cross-linking time of 1 hour.

$10 \mathrm{~mL}$ of phosphate buffer ( $\mathrm{pH} 7.4$ ). After 24 hours, the solution was filtered and the filtrate was analyzed for the drug content. The drug entrapment efficiency was calculated using the following formula: Practical drug content/ Theoretical drug content $\times 100$. The drug entrapment efficiency for batches $\mathrm{J} 1$ to $\mathrm{J} 15$ and $\mathrm{B} 1$ to $\mathrm{B} 9$ are reported in Tables 1 and 2, respectively.

\section{Particle Size and Swelling Index of Microspheres}

The particle size of the microspheres was determined by using optical microscopy method. ${ }^{22}$ Approximately 100 microspheres were counted for particle size using a calibrated optical microscope (Labomed CX RIII, Ambala, India).

For estimating the swelling index, the microspheres $(\sim 100)$ were suspended in $5 \mathrm{~mL}$ of simulated gastric fluid USP (pH 1.2). ${ }^{23}$ The particle size was monitored by microscopy technique every 1 hour using an optical microscope (Labomed CX RIII). The increase in particle size of the microspheres was noted for up to 8 hours, and the swelling index was calculated as per method described by Ibrahim. ${ }^{24}$ The swelling index for microspheres of batches B1 to $\mathrm{B} 9$ is reported in Table 2.

\section{In Vitro Wash-off Test for Microspheres}

The mucoadhesive properties of the microspheres were evaluated by in vitro wash-off test as reported by Lehr et al. ${ }^{25}$ A $1-\mathrm{cm}$ by $1-\mathrm{cm}$ piece of rat stomach mucosa was tied onto a glass slide (3-inch by 1-inch) using thread. Microspheres were spread $(\backsim 50)$ onto the wet, rinsed, tissue specimen, and the prepared slide was hung onto one of the groves of a USP tablet disintegrating test apparatus. The disintegrating test apparatus was operated such that the tissue specimen was given regular up and down movements in a beaker containing the simulated gastric fluid USP ( $\mathrm{pH} 1.2$ ). At the end of 30 minutes, 1 hour, and at hourly intervals up to 10 hours, the number of microspheres still adhering onto the tissue was counted. The results of in vitro wash-off test of batches B1 to B9 are shown in Tables 1 and 2 respectively.

\section{Drug Release Study}

The drug release study was performed using USP XXIV basket apparatus (Electrolab, TDT-06T, Mumbai, India) at $37^{\circ} \mathrm{C} \pm 0.5^{\circ} \mathrm{C}$ and at $50 \mathrm{rpm}$ using $900 \mathrm{~mL}$ of phosphate buffer $(\mathrm{pH} 7.4)$ as a dissolution medium $(\mathrm{n}=5)$ as per USP XXVI dissolution test prescribed for glipizide extended release tablets. ${ }^{21}$ Microspheres equivalent to $10 \mathrm{mg}$ of glipizide were used for the test. Five milliliters of sample solution was withdrawn at predetermined time intervals, filtered through a $0.45 \mu \mathrm{m}$ membrane filter, diluted suitably, and analyzed spectrophotometrically. An equal amount of fresh dissolution medium was replaced immediately after withdrawal of the test sample. Percentage drug dissolved at different time intervals was calculated using the Lambert-Beer's equation $(\mathrm{y}=0.0218 \mathrm{x}+$ $0.0147, \mathrm{R}^{2}=0.9979$ ) described above. The $\mathrm{t}_{80}$ was calcu- 


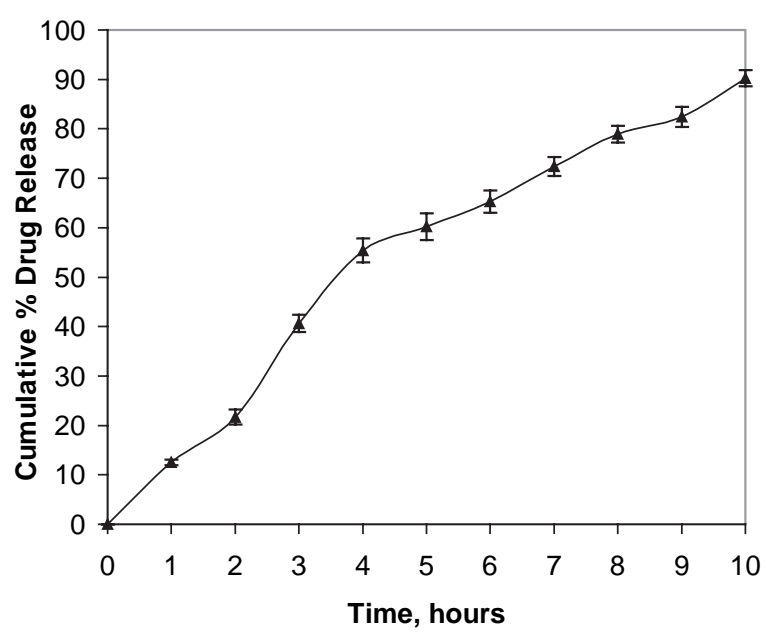

Figure 1. In vitro dissolution of glipizide from mucoadhesive microspheres of batch B7.

lated using the Weibull equation. ${ }^{26}$ The average values of $\mathrm{t}_{80}$ for batches B1 to B9 are mentioned in Table 2. The percentage drug release of batch B7 is shown in Figure 1.

\section{Scanning Electron Microscopy}

A scanning electron photomicrograph of drug-loaded chitosan mucoadhesive microspheres was taken. A small amount of microspheres was spread on glass stub. Afterwards, the stub containing the sample was placed in the scanning electron microscope (JSM 5610 LV SEM, JEOL, Datum Ltd, Tokyo, Japan) chamber. The scanning electron photomicrograph was taken at the acceleration voltage of $20 \mathrm{kV}$, chamber pressure of $0.6 \mathrm{~mm} \mathrm{Hg}$, original magnification $\times 800$. The photomicrograph is depicted in Figure 2 .

\section{Factorial Design}

A statistical model incorporating interactive and polynomial terms was used to evaluate the responses:

$$
Y=b_{0}+b_{1} X_{1}+b_{2} X_{2}+b_{12} X_{1} X_{2}+b_{11} X_{1}^{2}+b_{22} X_{2}^{2},
$$

where, $Y$ is the dependent variable, $b_{0}$ is the arithmetic mean response of the 9 runs, and $b_{\mathrm{i}}$ is the estimated coefficient for the factor $X_{\mathrm{i}}$. The main effects $\left(X_{1}\right.$ and $\left.X_{2}\right)$ represent the average result of changing one factor at a time from its low to high value. The interaction terms $\left(X_{1} X_{2}\right)$ show how the response changes when 2 factors are simultaneously changed. The polynomial terms $\left(X_{1}^{2}\right.$ and $\left.X_{2}^{2}\right)$ are included to investigate nonlinearity.

\section{In Vivo Study}

In vivo evaluation studies for glipizide mucoadhesive microspheres were performed on normal healthy Wistar rats weighing 250 to $300 \mathrm{~g}$ each. The approval of the Insti- tutional Animal Ethics Committee was obtained before starting the study. The study was conducted in accordance with standard institutional guidelines. Two groups of Wistar rats (5 in each group) that were fasted (with water) at least 12 hours before the experiments were used for the study. Before drug administration, a blood sample as a control was taken from each rat from behind the eyeball through the angle of ocular cavity using small capillary tubes. The blood glucose level for the control and test samples was determined using the glucose-measuring instrument Medisence (Abbott Laboratories, Bedford, MA). The instrument was self-calibrated, and the samples were allowed to dry before the results were read to avoid contamination of the lens. Pure glipizide and mucoadhesive microspheres of glipizide were administered orally to each group using stomach intubations. A dose of $800 \mu \mathrm{g} / \mathrm{kg}$ of glipizide was administrated in a suspension form (freshly prepared) for each rat. Blood samples were collected at predetermined time at 1-hour intervals up to 24 hours, and the blood glucose level was performed as per method described earlier. The percentage reduction in blood glucose level was measured and is depicted in Figure 3.

\section{RESULTS AND DISCUSSION}

\section{Preliminary Trials}

The mucoadhesive microspheres of chitosan were prepared by simple emulsification phase separation technique. Chitosan was selected as a polymer for the preparation of mucoadhesive microspheres owing to its biodegradable and mucoadhesive properties. Different concentrations of acetic acid from $1 \% \mathrm{vol} / \mathrm{vol}$ to $6 \% \mathrm{vol} / \mathrm{vol}$ were used for preparing the polymer solution, but no significant effect of concentration of acetic acid was observed on percentage mucoadhesion or drug entrapment efficiency, therefore $1 \% \mathrm{vol} / \mathrm{vol}$ of acetic acid was used. This finding could be owing to good solubility of chitosan in acetic acid.

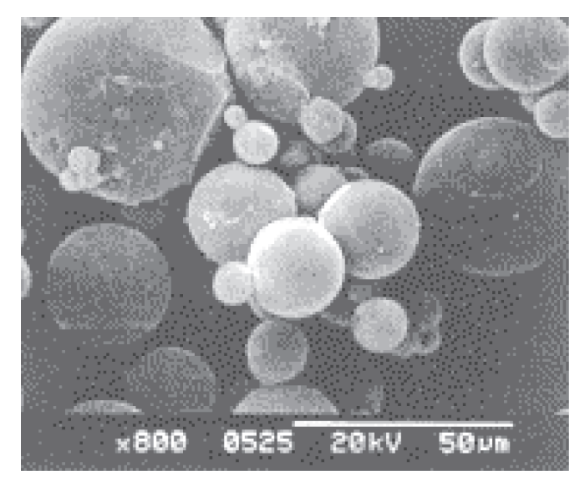

Figure 2. Scanning electron photomicrograph of glipizide-loaded chitosan mucoadhesive microspheres (batch B7). The parameters of scanning electron microscopy were acceleration voltage of $20 \mathrm{kV}$, chamber pressure of $0.6 \mathrm{~mm} \mathrm{Hg}$, original magnification $\times 800$. 


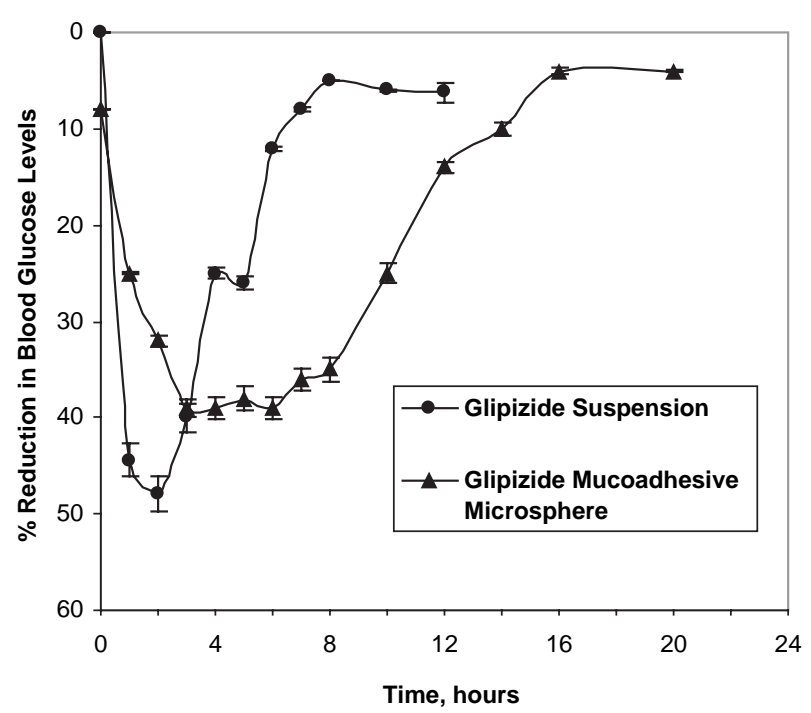

Figure 3. Percentage reduction in blood glucose levels following oral administration of glipizide (-๑) and its mucoadhesive microspheres (- $\mathbf{\Delta}-)$ in Wistar rats.

One of the important factors related to microspheres as reported by Lee et $\mathrm{al}^{27}$ is the viscosity of the polymer solution. Polymer concentrations of $0.5 \%, 1 \%$, and $2 \% \mathrm{wt} / \mathrm{vol}$ were selected for preliminary trials. Flake formation was observed when chitosan concentration was used at a level of $0.5 \%$, whereas maximum sphericity was observed at the $1 \%$ level. The chitosan solution was found to be too viscous to pass through the syringe when used at the $2 \%$ level. Therefore $1 \% \mathrm{wt} / \mathrm{vol}$ of chitosan in $1 \% \mathrm{vol} / \mathrm{vol}$ acetic acid was found to be the optimum concentration for the polymer solution. A 1:1 mixture of heavy and light liquid paraffin was found to be suitable as the dispersion medium. The addition of $0.2 \% \mathrm{wt} / \mathrm{vol}$ of DOSS to the dispersion medium was found to be essential to minimize aggregation of microspheres.

Batches J1 to J15 were prepared to study the effect of the volume of cross-linking agent (glutaraldehyde), time for cross-linking, and stirring speed on the percentage mucoadhesion, drug entrapment efficiency, and characteristics of the microspheres.

The volume of glutaraldehyde was varied from 10 to 60 $\mathrm{mL}$. Discrete spherical microspheres were obtained using 40,50 , and $60 \mathrm{~mL}$ of glutaraldehyde (batches $\mathrm{J} 7$ to J15).
Batches J1 to J6 prepared using 10 and $20 \mathrm{~mL}$ of glutaraldehyde yielded irregular microspheres. The higher amount of glutaraldehyde appears to favor the cross-linking reaction, and hence spherical free-flowing microspheres were obtained. The microspheres of batches J7 to J15 also showed significant effect on the percentage mucoadhesion and drug entrapment efficiency (Table 1). Microspheres of batches $\mathrm{J} 7$ to $\mathrm{J} 9$ prepared using $40 \mathrm{~mL}$ of glutaraldehyde showed good percentage mucoadhesion, but drug entrapment efficiency was below $63 \%$. Batches J10 to J12 also showed good mucoadhesion as well as $70 \%$ drug entrapment efficiency. In the microspheres of batches J13 to J15, the drug entrapment efficiency was above $68 \%$, but mucoadhesion decreased. The decrease in mucoadhesion could possibly be attributed to the greater amount of crosslinking agent giving a more rigid cross-linked polymer whose adhesion is decreased. Thus, we can conclude that $50 \mathrm{~mL}$ of glutaraldehyde was the optimum amount. Increase in the cross-linking time (1 to 3 hours) in batches $\mathrm{J} 1$ to J15 inversely affected the percentage mucoadhesion. The cross-linking polymer probably becomes more rigid and thus mucoadhesiveness decreases. The cross-linking time did not have a significant effect on the percentage drug entrapment efficiency.

On the basis of the preliminary trials a $3^{2}$ full factorial design was employed to study the effect of independent variables (ie, polymer-to-drug ratio $\left[X_{1}\right]$ and the stirring speed $\left[X_{2}\right]$ ) on dependent variables percentage mucoadhesion, $t_{80}$, drug entrapment efficiency, and swelling index. The results depicted in Table 2 clearly indicate that all the dependent variables are strongly dependent on the selected independent variables as they show a wide variation among the 9 batches (B1 to B9). The fitted equations (full models) relating the responses (ie, percentage mucoadhesion, $t_{80}$, drug entrapment efficiency, and swelling index) to the transformed factor are shown in Table 3 . The polynomial equations can be used to draw conclusions after considering the magnitude of coefficient and the mathematical sign it carries (ie, positive or negative). The high values of correlation coefficient (Table 3 ) for the dependent variables indicate a good fit. The equations may be used to obtain estimates of the response since small error of variance was noticed in the replicates.

Table 3. Summary of Results of Regression Analysis

\begin{tabular}{lcccccrr}
\hline Coefficient & $\mathbf{b}_{\mathbf{0}}$ & $\mathbf{b}_{\mathbf{1}}$ & $\mathbf{b}_{\mathbf{2}}$ & $\mathbf{b}_{\mathbf{1 1}}$ & $\mathbf{b}_{\mathbf{2 2}}$ & $\mathbf{b}_{\mathbf{1 2}}$ & $\mathbf{R}^{\mathbf{2}}$ \\
\hline \% Mucoadhesion & 65.22 & 12.67 & -6.17 & -1 & -7.33 & 1.17 & 0.9956 \\
$\mathrm{t}_{80}$ & 241.66 & 103.73 & -11.61 & -20.0656 & 101.62 & -14.84 & 0.9621 \\
Drug entrapment efficiency & 68.17 & 11.0 & -2.97 & -0.475 & -7.0 & -0.30 & 0.9981 \\
Swelling index & 1.08 & 0.24 & -0.07 & -0.03 & -0.02 & 0.01 & 0.9907 \\
\hline
\end{tabular}




\section{Factorial Equation for Percentage Mucoadhesion and Swelling Index}

The in vitro wash-off test for percentage mucoadhesion after 1 hour varied from 51 to 78 and showed good correlation coefficient (0.9956). Results of the equation indicate that the effect of $X_{1}$ (polymer-to-drug ratio) is more significant than $X_{2}$ (stirring speed). Moreover, stirring speed had a negative effect on the percentage mucoadhesion (ie, as the stirring speed increased, the percentage mucoadhesion decreased). This finding may be attributed to the change in particle size that affects mucoadhesion. Similar results were obtained for swelling index. The amount of polymer directly affected the solvent transfer rate; thus, as the polymer concentration increased the swelling index also increased. The swelling index varied from 0.866 to 1.423 and showed good correlation coefficient (0.9907). Thus, we can conclude that the amount of polymer and stirring speed directly affects the percentage mucoadhesion and swelling index.

\section{Factorial Equation for Drug Entrapment Efficiency and $t_{80}$}

The drug entrapment efficiency and $t_{80}$ are important variables for assessing the drug loading capacity of microspheres and their drug release profiles, thus suggesting the amount of drug availability at site. These parameters are dependent on the process of preparation, physicochemical properties of drug, and formulation variables. The drug entrapment efficiency varied from $47 \%$ to $76 \%$ and showed good correlation coefficient (0.9981). Results of the equation indicate that the effect of $X_{1}$ (polymer-to-drug ratio) is more significant than $X_{2}$ (stirring speed). Moreover, stirring speed had a negative effect on drug entrapment efficiency (ie, as the stirring speed increased, the particle size decreased, and thus drug entrapment efficiency decreased).

The mucoadhesive microspheres of all the batches of the factorial design were spherical and free flowing. They ranged in particle size from 48 to $96 \mu \mathrm{m}$. Results depicted in Table 3 indicate that the percentage drug released in vitro is highly dependent on the polymer-to-drug ratio and the stirring speed. The stirring speed has a negative effect on $t_{80}$ because as the particle size increases the drug released decreases. Higher levels of polymer-to-drug ratio favor the cross-linking reaction and thus higher $t_{80}$ is obtained. Batch B7 exhibited a high $t_{80}$ of 476 minutes and seems to be a promising candidate for achieving drug release up to 12 hours. The drug release profile of batch B7 is shown in Figure 1. The figure reveals that drug release rate was slowed after 4 hours. The study focus was the preparation of mucoadhesive microspheres, thus the microspheres of batch B7 were also evaluated in simulated gas- tric fluid USP ( $\mathrm{pH}$ 1.2). The results indicated that no significant difference was observed between dissolution profiles at $\mathrm{pH} 7.4$ and $\mathrm{pH} 1.2$ as the $f_{2}$ value was 65.2 . The particle size of microspheres of batch B7 was $96 \mu \mathrm{m}$. The photomicrograph of batch B7 is depicted in Figure 2, which indicates good sphericity of the microspheres. The dissolution data of batch B7 were further analyzed to ascertain the mechanism of drug release. ${ }^{28}$ The release profile fitted best to the Weibull equation $(F=9.05)$. The value of the correlation coefficient was found to be 0.987 . The values of slope and intercept were found to be 1.22 and -3.06 , respectively.

\section{In Vivo Study}

In vivo efficiency of the optimized batch B7 was performed in healthy normal Wistar rats by measuring the hypoglycemic effect produced after oral administration. The drug was administered at a dose equivalent to $800 \mu \mathrm{g} /$ $\mathrm{kg}$ pure glipizide, and glipizide mucoadhesive microspheres were used for the study. Pure glipizide drug was administered in a suspension form at the same dose. When pure glipizide suspension was administered, a rapid reduction in blood glucose levels was observed and maximum reduction of $48 \%$ was observed within 2 hours after oral administration. Blood glucose levels were recovered rapidly to the normal level within 8 hours (Figure 3). In the case of glipizide mucoadhesive microspheres, the reduction in blood glucose levels was slow and reached maximum reduction within 4 hours after oral administration. This reduction in blood glucose levels was sustained over longer periods of time (12 hours). Kahn and Shechter ${ }^{29}$ have suggested that a $25 \%$ reduction in blood glucose levels is considered a significant hypoglycemic effect. Significant hypoglycemic effect $(25 \%)$ was maintained only from 0.5 to 5 hours after oral administration of glipizide, whereas in the case of mucoadhesive glipizide microspheres, significant hypoglycemic effect $(25 \%)$ was maintained for a period of 2 to 12 hours. The sustained hypoglycemic effect observed over a longer period of time in the case of mucoadhesive microspheres is due to the slow release and absorption of glipizide over longer periods of time. Glipizide sustained release formulation is significantly more effective than the immediate release formulation of glipizide in reducing fasting plasma glucose levels and side effects. ${ }^{30}$ Formulation of glipizide as mucoadhesive sustained release dosage form could also exhibit a decrease in side effects.

\section{CONCLUSION}

The results of a $3^{2}$ full factorial design revealed that the polymer-to-drug ratio and stirring speed significantly affected the dependent variables percentage mucoadhesion, 


\section{AAPS PharmSciTech 2005; 6 (1) Article 10 (http://www.aapspharmscitech.org).}

$t_{80}$ drug entrapment efficiency, and swelling index. The microspheres of the best batch exhibited a high percentage mucoadhesion of $78 \%$ after 1 hour, $75 \%$ drug entrapment efficiency, and swelling index of 1.42 . The $t_{80}$ of 476 minutes indicates that the mucoadhesive microspheres of glipizide could sustain the release of the drug for more than 12 hours. The in vivo study demonstrated significant hypoglycemic activity of the mucoadhesive microspheres of glipizide.

\section{REFERENCES}

1. Woo BH, Jiang G, Jo YW, DeLuca PP. Preparation and characterization of a composite PLGA and poly (acryloyl hydroxymethyl starch) microsphere system for protein delivery. Pharm Res. 2001;18:1600-1606.

2. Capan Y, Jiang G, Giovagnoli S, DeLuca PP. Preparation and characterization of poly (D,L-lactide-co-glycolide) microsphere for controlled release of human growth hormone. AAPS PharmSciTech. 2003;4:E28.

3. Gohel MC, Amin AF. Formulation optimization of controlled release diclofenac sodium microspheres using factorial design. $J$ Control Release. 1998;51:115-122.

4. Vasir JK, Tambwekar K, Garg S. Bioadhesive microspheres as a controlled drug delivery system. Int J Pharm. 2003;255:13-32.

5. Ikeda K, Murata K, Kobayashi M, Noda K. Enhancement of bioavailability of dopamine via nasal route in beagle dogs. Chem Pharm Bull (Tokyo). 1992;40:2155-2158.

6. Nagai T, Nishimoto Y, Nambu N, Suzuki Y, Sekine K. Powder dosage form of insulin for nasal administration. $J$ Control Release. $1984 ; 1: 15-22$.

7. Ilium L, Farraj NF, Critchley H, Davis SS. Nasal administration of gentamicin using a novel microsphere delivery system. Int J Pharm. 1988;46:261-265.

8. Schaefer MJ, Singh J. Effect of isopropyl myristic acid ester on the physical characteristics and in vitro release of etoposide from PLGA microspheres. AAPS PharmSciTech. 2000;1:E32.

9. Rao SB, Sharma CP. Use of chitosan as biomaterial: studies on its safety and hemostatic potential. J Biomed Mater Res. 1997;34:21-28.

10. Lehr CM, Bouwstra JA, Schacht EH, Junginger HE. In vitro evaluation of mucoadhesive properties of chitosan and some other natural polymers. Int J Pharm. 1992;78:43-48.

11. Henriksen I, Green KL, Smart JD, Smistad G, Karlsen J. Bioadhesion of hydrated chitosans: an in vitro and in vivo study. Int J Pharm. 1996;145:231-240.

12. Chowdary KPR, Rao YS. Design and in vitro and in vivo evaluation of mucoadhesive microcapsules of glipizide for oral controlled release: a technical note. AAPS PharmSciTech. 2003;4:E39.

13. Thanoo BC, Sunny MC, Jayakrishnan A. Cross-linked chitosan microspheres: preparation and evaluation as a matrix for the controlled release of pharmaceuticals. J Pharm Pharmacol. 1992;44:283-286.
14. Hari PR, Chandy T, Sharma CP. Chitosan/calcium alginate microcapsules for intestinal delivery of nitrofurantoin. J Microencapsul. 1996;13:319-329.

15. Liu LS, Liu SQ, Ng SY, Froix M, Heller J. Controlled release of interleukin 2 for tumour immunotherapy using alginate/chitosan porous microspheres. J Control Release. 1997;43:65-74.

16. Patel JK, Bodar MS, Amin AF, Patel MM. Formulation and optimization of mucoadhesive microspheres of metoclopramide. Ind J Pharm Sci. 2004;66:300-305.

17. Dubey RR, Parikh RH. Two-stage optimization process for formulation of chitosan microspheres. AAPS PharmSciTech. 2004;5:E5.

18. Foster RH, Plosker GL. Glipizide: a review of the pharmacoeconomic implications of the extended-release formulation in type 2 diabetes mellitus. Pharmacoeconomics. 2000;18:289-306.

19. Thombre AG, Denoto AR, Gibbes DC. Delivery of glipizide from asymmetric membrane capsules using encapsulated excipients. J Control Release. 1999;60:333-341.

20. Chowdary KPR, Balatripura G. Design and in vitro evaluation of mucoadhesive controlled release oral tablets of glipizide. Ind J Pharm Sci. 2003;65:591-594.

21. The United States Pharmacopeial Convention. The United States Pharmacopeia. XXVI. Rockville, MD: The United States Pharmacopeial Convention, Inc; 2003:859.

22. Eugene L. Milling. In: Lachman L, Liberman HA. The Theory and Practice of Industrial Pharmacy. 2nd ed. Mumbai, India: Varghese Publishing House; 1991:26-27.

23. The United States Pharmacopeial Convention. The United States Pharmacopeia. XXVI. Rockville, MD: The United States Pharmacopeial Convention, Inc; 2003:2528.

24. El-Gibaly I. Development and in vitro evaluation of novel floating chitosan microcapsules for oral use: comparison with non-floating chitosan microspheres. Int J Pharm. 2002;249:7-21.

25. Lehr CM, Bowstra JA, Tukker JJ, Junginger HE. Intestinal transit of bioadhesive microspheres in an in situ loop in the rat. $J$ Control Release. 1990;13:51-62.

26. Nelson KG, Wang LY. Determination of time course of tablet disintegration II: method using continuous functions. J Pharm Sci. 1961;67:86-89

27. Lee JW, Park JH, Robinson JR. Bioadhesive-based dosage forms: the next generation. J Pharm Sci. 2000;89:850-866.

28. Bamba M, Puisieux F, Marty JP, Carstensen JT. Release mechanisms in gelforming sustained release preparations. Int J Pharm. 1979;2:307-315.

29. Kahn CR, Shechter Y. Oral hypoglycemic agents and the pharmacology of the endocrine pancreas. In: Theodore WR, Alan SN, Taylor P, Gilman AG, eds. Goodman and Gilman's The Pharmacological Basis of Therapeutics. 8th ed. New York, NY: McGraw-Hill; 1991:1461-1495.

30. Berelowitz M, Fischette C, Cefelu W, Schade DS Sutfin T, Kourides IA. Comparative efficacy of a once-daily controlled-release formulation of glipizide and immediate-release glipizide in patients with NIDDM. Diabetes Care. 1994;17:1460-1464. 